ANNALES

POLONICI MATHEMATICI

$87(2005)$

\title{
The Łojasiewicz gradient inequality in a neighbourhood of the fibre
}

\author{
by Janusz GwoźdzIEwicz (Kielce) and StanisŁaw Spodzieja (Łódź)
}

\begin{abstract}
Some estimates of the Łojasiewicz gradient exponent at infinity near any fibre of a polynomial in two variables are given. An important point in the proofs is a new Charzyński-Kozłowski-Smale estimate of critical values of a polynomial in one variable.
\end{abstract}

1. Introduction. In this paper, effective estimates relating to the Eojasiewicz gradient inequality at infinity for polynomials in two variables are given. To achieve them, we prove an estimate for critical values of a polynomial in one variable (Theorem 2.1), which is a version of the CharzyńskiKozłowski [2] and Smale [24] theorems. Namely, if $P: \mathbb{C} \rightarrow \mathbb{C}$ is a polynomial of degree $d>1, \varphi_{1}, \ldots, \varphi_{d} \in \mathbb{C}$ and $\xi_{1}, \ldots, \xi_{d-1} \in \mathbb{C}$ are all roots of $P$ and of its derivative $P^{\prime}$, respectively, and $a$ is the leading coefficient of $P$, then

$$
\min _{1 \leq k \leq d-1}\left|P\left(\xi_{k}\right)\right| \leq 4|a|^{-1 /(d-1)}\left[\min _{1 \leq i \leq d}\left|P^{\prime}\left(\varphi_{i}\right)\right|\right]^{d /(d-1)} .
$$

The first result (Theorem 3.1) on the Łojasiewicz inequality is the following generalisation of the Bochnak-Łojasiewicz inequality ([1, Lemma 2], [25, Theorem 1]); for a polynomial $f: \mathbb{C}^{n} \rightarrow \mathbb{C}$, there exist $C, \varepsilon>0$ such that

$$
|f(z)| \leq \varepsilon \Rightarrow|z| \operatorname{grad} f(z)|\geq C| f(z) \mid,
$$

where $|\cdot|$ is a norm in $\mathbb{C}^{n}$.

From the above two inequalities, we obtain an estimate of the Eojasiewicz gradient exponent in a neighbourhood of the bifurcation fibre of a polynomial. By definition, the Łojasiewicz exponent at infinity $\mathcal{L}_{\infty}(F \mid X)$ of a polynomial mapping $F: \mathbb{C}^{n} \rightarrow \mathbb{C}^{m}$ on an unbounded set $X \subset \mathbb{C}^{n}$ is the

2000 Mathematics Subject Classification: 14E05, 14R25, 30C15.

Key words and phrases: polynomial, Łojasiewicz inequality, bifurcation point.

Research of J. Gwoździewicz was partially supported by KBN Grant 2 PO3A 01522.

Research of S. Spodzieja was partially supported by KBN Grant 2 PO3A 00718. 
best exponent $\nu$ in the inequality

$$
|F(z)| \geq C|z|^{\nu} \quad \text { as } z \in X, z \rightarrow \infty,
$$

for some constant $C>0$. In the case $F=\operatorname{grad} f$, where $f$ is a polynomial in two variables, and $X=\left\{z \in \mathbb{C}^{2}:|f(z)|<\varepsilon\right\}$ is a neighbourhood of $f^{-1}(0), \mathcal{L}_{\infty}(\operatorname{grad} f \mid X)$ is equal to the exponent $\mathcal{L}_{\infty, 0}(f)$ considered by Ha [8] and by Chądzyński and Krasiński [5], provided $\varepsilon>0$ is sufficiently close to 0. It is shown in Theorem 4.2 and Corollary 6.1 (cf. [8, Theorem 1.3.2], [12, Theorem 3.1]) that if $0 \in \mathbb{C}$ is a bifurcation point of $f$ at infinity (see Section 6 ), and $d=\operatorname{deg} f>2$, then for any neighbourhood $X$ of $f^{-1}(0)$,

or equivalently

$$
\mathcal{L}_{\infty}(\operatorname{grad} f \mid X) \leq-1-\frac{1}{d-2}
$$

$$
\mathcal{L}_{\infty}(f, \operatorname{grad} f)<0 .
$$

Estimate $(*)$ is sharp as regards the degree of $f$ (see Remark 6.3). In particular, we obtain the following result of Gwoździewicz and Płoski (see [7, Theorem 5.2]): if the bifurcation set of $f$ at infinity is nonempty, then $\mathcal{L}_{\infty}(\operatorname{grad} f) \leq-1-1 /(d-2)$ (Corollary 6.2$)$.

Theorem 4.2 gives a sharper version of the Malgrange condition (condition $(\mathrm{m})$ in [15]), namely the inequality

$$
|z|^{\alpha}|\operatorname{grad} f(z)| \geq \eta \quad \text { as } z \rightarrow \infty \text { and } f(z) \rightarrow 0
$$

does not depend on the choice of $\alpha$ such that $0 \leq \alpha<1+1 /(d-2)$ (Proposition 5.1).

In Theorem 7.5 we prove the following separation condition of $\operatorname{grad} f$ and $f$ (introduced by Płoski and Tworzewski in [20], see also [25]):

$$
|f(z)| \leq \varepsilon \Rightarrow|\operatorname{grad} f(z)| \geq C|f(z)|^{q}
$$

for some $C, \varepsilon, q>0$. Moreover one can take $q=(d-1)^{2}$. In the general case, i.e. $f: \mathbb{C}^{n} \rightarrow \mathbb{C}, n>2$, condition (PT) may not be satisfied (see [25, Remark 2] and [21, Remark 9.1]). The description of polynomials for which (PT) holds is given in Remark 7.6.

2. The Charzyński-Kozłowski-Smale Theorem. In this section we give a version of the Charzyński-Kozłowski (see [2, Theorem 3]) and Smale (see [24, p. 33]) Theorem.

Theorem 2.1. Let $P: \mathbb{C} \rightarrow \mathbb{C}$ be a polynomial of degree $d>1$, and let $\varphi_{1}, \ldots, \varphi_{d} \in \mathbb{C}$ and $\xi_{1}, \ldots, \xi_{d-1} \in \mathbb{C}$ be all roots of $P$ and $P^{\prime}$, respectively. Then

$$
\min _{1 \leq k \leq d-1}\left|P\left(\xi_{k}\right)\right| \leq 4 \min _{i \neq j}\left|\varphi_{i}-\varphi_{j}\right|\left|P^{\prime}\left(\varphi_{i}\right)\right|
$$


In particular,

$$
\min _{1 \leq k \leq d-1}\left|P\left(\xi_{k}\right)\right| \leq 4|a|^{-1 /(d-1)}\left[\min _{1 \leq i \leq d}\left|P^{\prime}\left(\varphi_{i}\right)\right|\right]^{d /(d-1)},
$$

where $a \in \mathbb{C}$ is the leading coefficient of $P$.

Proof. If $A \subset \mathbb{C}$ and $b \in \mathbb{C}$, we put $A-b=\{a-b: a \in A\}$ and $b A=\{a b: a \in A\}$. For $a \in \mathbb{C}$ and $r>0$, we denote by $D(a, r)$ the disc with centre at $a$ and radius $r$.

Let

$$
R=\min _{1 \leq k \leq d-1}\left|P\left(\xi_{k}\right)\right|
$$

It suffices to consider the case $R>0$. Then obviously $\varphi_{i} \neq \varphi_{j}$ for $i \neq j$. Let $G=P^{-1}(D(0, R))$. Since $\left.P\right|_{G}: G \rightarrow D(0, R)$ has no critical values, it is a $d$-sheeted covering. As $D(0, R)$ is a simply connected domain, $G=$ $G_{1} \cup \cdots \cup G_{d}$, where $G_{1}, \ldots, G_{d}$ are domains such that $\left.P\right|_{G_{i}}: G_{i} \rightarrow D(0, R)$ is a biholomorphism, $i=1, \ldots, d$. Write $f_{i}=\left(\left.P\right|_{G_{i}}\right)^{-1}: D(0, R) \rightarrow G_{i}$. We may renumber $f_{i}$ so that $\varphi_{i}=f_{i}(0), i=1, \ldots, d$. Take any $i \in\{1, \ldots, d\}$ and put

$$
g_{i}(w)=\frac{1}{R f_{i}^{\prime}(0)}\left[f_{i}(w R)-\varphi_{i}\right], \quad w \in D(0,1) .
$$

Each $g_{i}$ is an injective holomorphic function such that $g_{i}(0)=0$ and $g_{i}^{\prime}(0)=1$. Therefore, by the Koebe Theorem (see [10]), $D(0,1 / 4) \subset g_{i}(D(0,1))$. In consequence,

$$
D\left(0, R\left|f_{i}^{\prime}(0)\right| / 4\right) \subset R f_{i}^{\prime}(0) g_{i}(D(0,1))=f_{i}(D(0, R))-\varphi_{i} .
$$

Hence,

$$
D\left(\varphi_{i}, R\left|f_{i}^{\prime}(0)\right| / 4\right) \subset f_{i}(D(0, R))=G_{i} .
$$

Since $\varphi_{j} \notin G_{i}$ for $i \neq j$, by the above we have

$$
\frac{R}{4\left|P^{\prime}\left(\varphi_{i}\right)\right|}=\frac{R\left|f_{i}^{\prime}(0)\right|}{4} \leq\left|\varphi_{i}-\varphi_{j}\right|, \quad i \neq j .
$$

Hence (1) follows. From (1) we see that

$$
\min _{1 \leq k \leq d-1}\left|P\left(\xi_{k}\right)\right| \leq 4\left|\varphi_{i}-\varphi_{j}\right|\left|P^{\prime}\left(\varphi_{i}\right)\right| \quad \text { for any } j \neq i
$$

Thus, from $P^{\prime}\left(\varphi_{i}\right)=a \prod_{j \neq i}\left(\varphi_{i}-\varphi_{j}\right)$, we deduce (2).

The inequality (1) cannot be improved, except for the constant 4 . Namely we have

Proposition 2.2. Let $P: \mathbb{C} \rightarrow \mathbb{C}$ be a polynomial of degree $d>1$, and let $\varphi_{1}, \ldots, \varphi_{d} \in \mathbb{C}$ and $\xi_{1}, \ldots, \xi_{d-1} \in \mathbb{C}$ be all roots of $P$ and $P^{\prime}$, respectively. Then

$$
\frac{2^{d-2}}{3^{d}} \min _{i \neq j}\left|\varphi_{i}-\varphi_{j}\right|\left|P^{\prime}\left(\varphi_{i}\right)\right| \leq \min _{1 \leq k \leq d-1}\left|P\left(\xi_{k}\right)\right|
$$


Proof. Let $r=\min _{i \neq j}\left|\varphi_{i}-\varphi_{j}\right|\left|P^{\prime}\left(\varphi_{i}\right)\right|$. Under the notation of the proof of Theorem 2.1, it suffices to consider the case $R>0$. For any $i \in\{1, \ldots, d\}$, let $j_{i} \in\{1, \ldots, d\}$ be such that $\left|\varphi_{i}-\varphi_{j_{i}}\right|=\min _{i \neq j}\left|\varphi_{i}-\varphi_{j}\right|$, and let $r_{i}=$ $(1 / 2)\left|\varphi_{i}-\varphi_{j_{i}}\right|, M_{i}=(3 / 2)^{d-1} r_{i}\left|P^{\prime}\left(\varphi_{i}\right)\right|$. Set $D_{i}=D\left(\varphi_{i}, r_{i}\right), i=1, \ldots, d$. Then for any $z \in \bar{D}_{i}$ we easily obtain

$$
|P(z)|=\left|z-\varphi_{i}\right||a| \prod_{j \neq i}\left|z-\varphi_{j}\right| \leq \frac{1}{2}\left|\varphi_{i}-\varphi_{j_{i}}\right||a| \prod_{j \neq i}\left(\frac{3}{2}\left|\varphi_{i}-\varphi_{j}\right|\right)=M_{i} .
$$

Hence, by [23, Ch. VII, Theorem 12.7],

$$
D\left(0, \frac{r_{i}^{2}\left|P^{\prime}\left(\varphi_{i}\right)\right|^{2}}{6 M_{i}}\right) \subset P\left(D_{i}\right), \quad i=1, \ldots, d .
$$

For any $i \in\{1, \ldots, d\}$, from the definition of $r, r_{i}$ and $M_{i}$, we have

$$
\frac{2^{d-2}}{3^{d}} r \leq \frac{r_{i}^{2}\left|P^{\prime}\left(\varphi_{i}\right)\right|^{2}}{6 M_{i}}
$$

In consequence for $\widetilde{r}=2^{d-2} r / 3^{d}$, we obtain $D(0, \widetilde{r}) \subset P\left(D_{i}\right), i=1, \ldots, d$. Since $D_{i} \cap D_{j}=\emptyset$ for $i \neq j$, for any $w \in D(0, \widetilde{r})$ we have $\# P^{-1}(w)=d$. Summing up, $P$ has no critical values in $D(0, \widetilde{r})$, so $R \geq \widetilde{r}$ and we get (3).

REMARK 2.3. From Theorem 2.1 and Proposition 2.2, it is easy to prove the Kuo and Lu formula for the Eojasiewicz gradient exponent of a holomorphic function at zero [11] and the Ha formula for the Łojasiewicz gradient exponent at infinity of a polynomial [8] as is shown in [26].

3. The Kojasiewicz gradient inequality. Let $f: \mathbb{C}^{n} \rightarrow \mathbb{C}$ be a polynomial in $z_{1}, \ldots, z_{n}$ and let $\operatorname{grad} f=\left(\partial f / \partial z_{1}, \ldots, \partial f / \partial z_{n}\right): \mathbb{C}^{n} \rightarrow \mathbb{C}^{n}$. We will prove the following version of the Bochnak-Lojasiewicz inequality [1] (cf. the main result of [25]).

Theorem 3.1. Let $f: \mathbb{C}^{n} \rightarrow \mathbb{C}$ be a polynomial. Then there exist $C, \varepsilon>0$ such that

$$
|f(z)| \leq \varepsilon \Rightarrow|z||\operatorname{grad} f(z)| \geq C|f(z)| .
$$

We begin with a definition. A curve $\varphi:(r, \infty) \rightarrow \mathbb{R}^{k}, r \in \mathbb{R}$, is called meromorphic at $\infty$ if $\varphi$ is the sum of a Laurent series of the form

$$
\varphi(t)=\alpha_{p} t^{p}+\alpha_{p-1} t^{p-1}+\cdots, \quad \alpha_{i} \in \mathbb{R}^{k}, p \in \mathbb{Z} .
$$

If $\varphi \neq 0$, then we may assume that $\alpha_{p} \neq 0$. Then the number $p$ is called the degree of $\varphi$ and denoted by $\operatorname{deg} \varphi$. Additionally we put $\operatorname{deg} 0=-\infty$.

Proof of Theorem 3.1. As in [25], we use Hörmander's method. The Eojasiewicz inequality does not depend on a particular norm in $\mathbb{C}^{n}$, so we shall use the Euclidean norm $\|\cdot\|$. Assume to the contrary that for any $\varepsilon>0$ there exists $z \in \mathbb{C}^{n}$ such that $|f(z)| \leq \varepsilon$ and $\|\operatorname{grad} f(z)\|\|z\|<\varepsilon|f(z)|$. Then 
there exists $z^{0} \in \mathbb{C} \cup\{\infty\}$ such that $\left(z^{0}, 0\right)$ is an accumulation point of the semi-algebraic set

$$
X=\left\{(z, \varepsilon) \in \mathbb{C}^{n} \times \mathbb{R}: \varepsilon>0 \wedge|f(z)| \leq \varepsilon \wedge\|z\|\|\operatorname{grad} f(z)\|<\varepsilon|f(z)|\right\} .
$$

Thus, by the Curve Selection Lemma ([13, Lemma 3.1]), there exists a curve $\psi=\left(\varphi, \varphi_{n+1}\right):(r, \infty) \rightarrow X$ meromorphic at $\infty$, such that $\lim _{t \rightarrow \infty} \psi(t)=$ $\left(z^{0}, 0\right)$. Hence, $\operatorname{deg} \varphi_{n+1}<0$. By the definition of $X$ we have

$$
\operatorname{deg}((\operatorname{grad} f) \circ \varphi)+\operatorname{deg} \varphi \leq \operatorname{deg} \varphi_{n+1}+\operatorname{deg} f \circ \varphi<\operatorname{deg} f \circ \varphi
$$

and $\operatorname{deg} f \circ \varphi \neq 0$. This is impossible, because, for $\varphi=\left(\varphi_{1}, \ldots, \varphi_{n}\right)$, we get

$$
\begin{aligned}
\operatorname{deg} f \circ \varphi & =1+\operatorname{deg}(f \circ \varphi)^{\prime}=1+\operatorname{deg}\left(\sum_{i=1}^{n} \frac{\partial f}{\partial z_{i}}(\varphi) \varphi_{i}^{\prime}\right) \\
& \leq \operatorname{deg}((\operatorname{grad} f) \circ \varphi)+\operatorname{deg} \varphi .
\end{aligned}
$$

REMARK 3.2. From Theorem 3.1 we easily obtain the analogous Łojasiewicz inequality for a real polynomial $f: \mathbb{R}^{n} \rightarrow \mathbb{R}$.

4. The Łojasiewicz exponent of the gradient. Let us start from the precise definition of the Eojasiewicz exponent. Let $F: \mathbb{C}^{2} \rightarrow \mathbb{C}^{m}$ be a polynomial mapping, and let $X \subset \mathbb{C}^{2}$ be an unbounded set. Put

$$
N(F \mid X):=\left\{\nu \in \mathbb{R}: \exists_{A, B>0} \forall_{z \in X}\left(|z| \geq B \Rightarrow A|z|^{\nu} \leq|F(z)|\right)\right\} .
$$

By the Eojasiewicz exponent at infinity of $F$ on $X$ we mean $\mathcal{L}_{\infty}(F \mid X)=$ $\sup N(F \mid X)$ if $N(F \mid S) \neq \emptyset$, and $\mathcal{L}_{\infty}(F \mid X)=-\infty$ if $N(F \mid X)=\emptyset$. If $X=\mathbb{C}^{2}$, we write $\mathcal{L}_{\infty}(F)$ and call it the Lojasiewicz exponent at infinity of $F$.

Let $U \subset \mathbb{C}$ be a neighbourhood of infinity, i.e. the complement of a compact set. Analogously to the real case, a mapping $h: U \rightarrow \mathbb{C}^{m}$ is called meromorphic at infinity if $h$ is the sum of a Laurent series of the form

$$
h(t)=\alpha_{p} t^{p}+\alpha_{p-1} t^{p-1}+\cdots, \quad t \in U, \alpha_{i} \in \mathbb{C}^{m}, p \in \mathbb{Z} .
$$

If $m=1$, then $h$ is called a function meromorphic at infinity.

Throughout the remainder of this section, let $f: \mathbb{C}^{2} \rightarrow \mathbb{C}$ be a polynomial in $z=(x, y)$, and let $d=\operatorname{deg} f \geq 2$. Let $V=\left\{z \in \mathbb{C}^{2}: f(z)=0\right\}$ and

$$
V_{x}=\left\{z \in \mathbb{C}^{2}: \frac{\partial f}{\partial x}(z)=0\right\}, \quad V_{y}=\left\{z \in \mathbb{C}^{2}: \frac{\partial f}{\partial y}(z)=0\right\} \text {. }
$$

If $\operatorname{deg} f=\operatorname{deg}_{y} f$ then, by the Puiseux Theorem at infinity (see [3, Lemmas 4.1 and 4.2]), there exist $N \in \mathbb{Z}, N>0, a \in \mathbb{C}$, and functions $\varphi_{1}, \ldots, \varphi_{d}, \xi_{1}, \ldots, \xi_{d-1}: U \rightarrow \mathbb{C}$ meromorphic at infinity such that

$$
f\left(t^{N}, y\right)=a \prod_{i=1}^{d}\left(y-\varphi_{i}(t)\right), \quad \frac{\partial f}{\partial y}\left(t^{N}, y\right)=a d \prod_{k=1}^{d-1}\left(y-\xi_{k}(t)\right) \text {. }
$$


From (2) in Theorem 2.1 we immediately obtain (cf. [5, Theorem 3.3]).

Proposition 4.1. Under the above assumptions,

$$
\mathcal{L}_{\infty}\left(f \mid V_{y}\right) \leq \frac{d}{d-1} \mathcal{L}_{\infty}(\operatorname{grad} f \mid V) .
$$

Proof. It is easy to see that

$$
\mathcal{L}_{\infty}\left(f \mid V_{y}\right)=\frac{1}{N} \min _{1 \leq k \leq d-1} \operatorname{deg} f\left(t^{N}, \xi_{k}(t)\right)
$$

and

$$
\frac{1}{N} \min _{1 \leq i \leq d} \operatorname{deg} \frac{\partial f}{\partial y}\left(t^{N}, \varphi_{i}(t)\right) \leq \mathcal{L}_{\infty}(\operatorname{grad} f \mid V) .
$$

Hence and from (2) in Theorem 2.1 the assertion follows.

Let us state a generalisation of the Ha Theorem (cf. [8, Theorem 1.3.2], [12, Theorem 3.1], [5, Corollary 3.5]).

Theorem 4.2. For every polynomial $f: \mathbb{C}^{2} \rightarrow \mathbb{C}$ with $d=\operatorname{deg} f>2$, the following conditions are equivalent:

(i) $\mathcal{L}_{\infty}(f, \operatorname{grad} f)<0$,

(ii) $\mathcal{L}_{\infty}(f, \operatorname{grad} f) \leq-1 /(d-2)$,

(iii) $\mathcal{L}_{\infty}(\operatorname{grad} f \mid X) \leq-1-1 /(d-2)$ for any $X=\left\{z \in \mathbb{C}^{2}:|f(z)|<\varepsilon\right\}$, where $\varepsilon>0$.

Proof. The implication (iii) $\Rightarrow$ (i) follows from Theorem 3.1 , and (ii) $\Rightarrow$ (i) is trivial.

Assume (i). We prove (ii) and (iii). Since $\mathcal{L}_{\infty}(\operatorname{grad} f)$ and $\mathcal{L}_{\infty}(f, \operatorname{grad} f)$ do not depend on the choice of the coordinate system, after a linear change of coordinates one can assume that $\operatorname{deg} f=\operatorname{deg}_{x} f=\operatorname{deg}_{y} f$. Moreover one can assume that $f$ has no multiple factors and (4) holds. Take any $X=\left\{z \in \mathbb{C}^{2}:|f(z)|<\varepsilon\right\}$, where $\varepsilon>0$. By [4, Theorem 1] and (i),

$$
\begin{aligned}
\min \left\{\mathcal{L}_{\infty}(\operatorname{grad} f \mid V), \mathcal{L}_{\infty}\left(\left(f, \frac{\partial f}{\partial x}\right) \mid V_{y}\right), \mathcal{L}_{\infty}(\right. & \left.\left.\left(f, \frac{\partial f}{\partial y}\right) \mid V_{x}\right)\right\} \\
& =\mathcal{L}_{\infty}(f, \operatorname{grad} f)<0 .
\end{aligned}
$$

If $\mathcal{L}_{\infty}(\operatorname{grad} f \mid V)=\mathcal{L}_{\infty}(f, \operatorname{grad} f)$, then, by Proposition $4.1, \mathcal{L}_{\infty}\left(f \mid V_{y}\right)$ $<0$. So, by [7, Theorem 2.9],

$$
\mathcal{L}_{\infty}\left(f \mid V_{y}\right) \leq-\frac{1}{d-2}
$$

Moreover, there exists $k_{0} \in\{1, \ldots, d-1\}$ such that $(1 / N) \operatorname{deg} f\left(t^{N}, \xi_{k_{0}}(t)\right)=$ $\mathcal{L}_{\infty}\left(f \mid V_{y}\right)$, and, for some $R \geq r, Y=\left\{\left(t^{N}, \xi_{k_{0}}(t)\right) \in \mathbb{C}^{2}:|t| \geq R^{1 / N}\right\}$ is an 
unbounded subset of $V_{y} \cap X$. Therefore,

(6) $\mathcal{L}_{\infty}(\operatorname{grad} f \mid Y)=\frac{1}{N} \operatorname{deg} \frac{\partial f}{\partial x}\left(t^{N}, \xi_{k_{0}}(t)\right) \leq-1+\frac{1}{N} \operatorname{deg} f\left(t^{N}, \xi_{k_{0}}(t)\right)$

$$
=-1+\mathcal{L}_{\infty}\left(f \mid V_{y}\right)
$$

and (5) gives (ii) in this case. Moreover, by (6),

$$
\mathcal{L}_{\infty}(\operatorname{grad} f \mid X) \leq \mathcal{L}_{\infty}(\operatorname{grad} f \mid Y) \leq-1-\frac{1}{d-2}
$$

This gives (iii) in this case.

If $\mathcal{L}_{\infty}\left(f, \frac{\partial f}{\partial x} \mid V_{y}\right)=\mathcal{L}_{\infty}(f, \operatorname{grad} f)$, then $\mathcal{L}_{\infty}\left(f \mid V_{y}\right)<0$ and as above we deduce (ii) and (iii).

If $\mathcal{L}_{\infty}\left(f, \frac{\partial f}{\partial y} \mid V_{x}\right)=\mathcal{L}_{\infty}(f, \operatorname{grad} f)$, then, by exchanging the roles of $x$ and $y$, from the second case we obtain (ii) and (iii) in this case. This ends the proof.

REMARK 4.3. The omitted case $\operatorname{deg} f \leq 2$ in Theorem 4.2 is not essential. Indeed, for $\operatorname{deg} f=1$, the gradient of $f$ is a constant mapping. For $\operatorname{deg} f=2$, either $f$ is a square function of a linear polynomial or $\mathcal{L}_{\infty}(\operatorname{grad} f)>0$.

From the proof of Theorem 4.2 we obtain the following version of Theorem 3.4 in [5].

Corollary 4.4. If $\mathcal{L}_{\infty}(f, \operatorname{grad} f)<0$, then

$$
\mathcal{L}_{\infty}(\operatorname{grad} f \mid X)=\mathcal{L}_{\infty}(f, \operatorname{grad} f)-1,
$$

where $X=\left\{z \in \mathbb{C}^{2}:|f(z)|<\varepsilon\right\}$ and $\varepsilon>0$ is sufficiently close to 0 .

Proof. By Theorem 3.1 we have $\mathcal{L}_{\infty}(\operatorname{grad} f \mid X) \geq \mathcal{L}_{\infty}(f, \operatorname{grad} f)-1$. As in the proof of Theorem 4.2 we deduce $\mathcal{L}_{\infty}(\operatorname{grad} f \mid X) \leq \mathcal{L}_{\infty}(f, \operatorname{grad} f)-1$.

REMARK 4.5. We define

$$
l(f)=\frac{1}{N} \min _{i \neq j}\left(\operatorname{deg}\left(\varphi_{i}(t)-\varphi_{j}(t)\right)+\operatorname{deg} \frac{\partial f}{\partial y}\left(t^{N}, \varphi_{i}(t)\right)\right) .
$$

Then, by Theorem 2.1 , we obtain $\mathcal{L}_{\infty}\left(f \mid V_{y}\right)=l(f)$ and $\mathcal{L}_{\infty}\left(\operatorname{grad} f \mid V_{y}\right) \leq$ $l(f)-1$, so

$$
\mathcal{L}_{\infty}\left((f, \operatorname{grad} f) \mid V_{y}\right)=l(f)
$$

(cf. [7, B.2], [5, Proposition 2.3], [22, Proposition 2]). If $\mathcal{L}_{\infty}(f, \operatorname{grad} f)<0$, then by [5, Theorem 3.3] and Corollary 4.4,

$$
\mathcal{L}_{\infty}(f, \operatorname{grad} f)=\mathcal{L}_{\infty}\left(f \mid V_{y}\right)=l(f)
$$

(cf. [8, Theorem 1.4.1]).

5. The Fedoryuk and Malgrange conditions. From Theorem 4.2 we easily obtain the following proposition (cf. [8, Theorem 1.3.2], [12, Theorem $3.1])$. 
Proposition 5.1. Let $f: \mathbb{C}^{2} \rightarrow \mathbb{C}$ be a polynomial with $d=\operatorname{deg} f>2$, and let $\lambda \in \mathbb{C}$ and $0<\alpha<1+1 /(d-2)$. Then the following conditions are equivalent:

(i) there exist $\eta, R, \varepsilon>0$ such that

$$
|z| \geq R \wedge|f(z)-\lambda| \leq \varepsilon \Rightarrow|\operatorname{grad} f(z)| \geq \eta,
$$

(ii) there exist $\eta, R, \varepsilon>0$ such that

$$
|z| \geq R \wedge|f(z)-\lambda| \leq \varepsilon \Rightarrow|z|^{\alpha}|\operatorname{grad} f(z)| \geq \eta .
$$

Conditions (F) and (M) are called the Fedoryuk condition (see [6]) and the Malgrange condition (cf. [17]), respectively. Denote by $K_{\infty}(f)$ the set of $\lambda \in \mathbb{C}$ for which condition (F) fails, and by $\widetilde{K}_{\infty}(f)$ the set of $\lambda$ for which (M) with $\alpha=1$ fails.

Using Proposition 5.1 and the known fact that $\widetilde{K}_{\infty}(f)$ is finite (cf. [9, Theorem 1.1], see also [25, Corollary 4]) we deduce the following known fact.

Corollary 5.2. If $f: \mathbb{C}^{2} \rightarrow \mathbb{C}$ is a polynomial, then $K_{\infty}(f)$ is finite.

6. The Kojasiewicz exponent and bifurcation points. Let $f$ : $\mathbb{C}^{n} \rightarrow \mathbb{C}$ be a polynomial. The smallest set $B(f) \subset \mathbb{C}$ such that $f$ is a fibration outside $B(f)$ is called the bifurcation set of $f$. The smallest set $B_{\infty}(f) \subset \mathbb{C}$ such that $f$ is a fibration at infinity outside $B_{\infty}(f)$ is called the bifurcation set of $f$ at infinity. More precisely, $\lambda \notin B_{\infty}(f)$ if there exists a compact $H \subset \mathbb{C}^{n}$ such that $\left.f\right|_{\mathbb{C}^{n} \backslash H}: \mathbb{C}^{n} \backslash H \rightarrow \mathbb{C}$ is a trivial fibration over a neighbourhood $U \subset \mathbb{C}$ of $\lambda$. It is known that $B_{\infty}(f) \subset \widetilde{K}_{\infty}(f)$, and $B(f) \subset K_{0}(f) \cup \widetilde{K}_{\infty}(f)$, where $K_{0}(f)$ is the set of critical values of $f$ ([15, Lemma 1.2 and Remark 1.3]). In the case $n=2$ we have $B_{\infty}(f)=\widetilde{K}_{\infty}(f)$ ([15, Theorem 1.4]).

From Theorem 4.2 we immediately obtain (cf. [25, Corollary 4])

Corollary 6.1. Let $f: \mathbb{C}^{2} \rightarrow \mathbb{C}$ be a polynomial with $d=\operatorname{deg} f>2$. The following conditions are equivalent:

(i) $\lambda \in B_{\infty}(f)$,

(ii) $\mathcal{L}_{\infty}(f-\lambda$, grad $f)<0$,

(iii) $\mathcal{L}_{\infty}(f-\lambda$, grad $f) \leq-1 /(d-2)$,

(iv) $\mathcal{L}_{\infty}(\operatorname{grad} f \mid X) \leq-1-1 /(d-2)$ for any $X=\left\{z \in \mathbb{C}^{2}:|f(z)-\lambda|\right.$ $\leq \varepsilon\}, \varepsilon>0$.

Proof. Since $B_{\infty}(f)=\widetilde{K}_{\infty}(f)$, it follows that $\lambda \in B_{\infty}(f)$ if and only if there exists a sequence $\left\{z^{m}\right\} \subset \mathbb{C}^{2}$ with $z^{m} \rightarrow \infty$ such that $(f$, grad $f)\left(z^{m}\right) \rightarrow$ $(\lambda, 0,0)$. Thus $\lambda \in B_{\infty}(f)$ if and only if $\mathcal{L}_{\infty}(f-\lambda$, grad $f)<0$. This gives the equivalence (i) $\Leftrightarrow($ ii). The remaining equivalences immediately follow from Theorem 4.2. 
Corollary 6.1 implies the following result Gwoździewicz and Płoski (see [7, Theorem 5.2]):

Corollary 6.2. Let $f: \mathbb{C}^{2} \rightarrow \mathbb{C}$ be a polynomial with $d=\operatorname{deg} f>2$. If $B_{\infty}(f) \neq \emptyset$, then $\mathcal{L}_{\infty}(\operatorname{grad} f) \leq-1-1 /(d-2)$.

Proof. Since $B_{\infty}(f)=\widetilde{K}_{\infty}(f)$, it follows that there exist $\lambda \in \mathbb{C}$ and a sequence $\left\{z^{m}\right\} \subset \mathbb{C}^{2}$ with $z^{m} \rightarrow \infty$ such that $(f, \operatorname{grad} f)\left(z^{m}\right) \rightarrow(\lambda, 0,0)$. Thus $\mathcal{L}_{\infty}(f-\lambda$, grad $f)<0$, and by Corollary 6.1 we get the assertion.

REMARK 6.3. The estimate in Corollary 6.1(iv) cannot be improved as regards the degree. Indeed, for the polynomial $f(x, y)=y^{d}+x y^{d-1}+y$, $d>2$ and $\lambda=0$ equality holds (see [5, Example 4.11(b)]).

REMARK 6.4. In Theorem 4.2 and Corollary 6.1, we require no special form of the polynomial $f$. Under an additional assumption on the form of $f$, i.e. $f(x, y)=y^{d}+a_{1}(x) y^{d-1}+\cdots+a_{d}(x)$, where $d=\operatorname{deg} f>2$, one can obtain Corollary 6.1 and Theorem 4.2 from [7, Theorem 2.9] and [5, Theorem 3.3 and Corollary 3.5]. Indeed, if 0 is a bifurcation point of $f$ at infinity then one can prove that $\mathcal{L}_{\infty}(f, \operatorname{grad} f)=\mathcal{L}_{\infty}(f, \partial f / \partial y)$ and $\mathcal{L}_{\infty}(f, \partial f / \partial y) \leq-1 /(d-2)$. Hence, we easily obtain the assertions of the above-mentioned theorems.

7. Separation of the gradient. In this section we show that in the two-dimensional case the gradient of a polynomial and the polynomial are separated. We begin with definitions and general properties.

Let $F: \mathbb{C}^{n} \rightarrow \mathbb{C}^{m}$ be a polynomial mapping and let $f: \mathbb{C}^{n} \rightarrow \mathbb{C}$ be a polynomial. We say that $F$ and $f$ are separated at infinity (see [20]) if there exist $C, R>0$ and $q \in \mathbb{R}$ such that

$$
|f(z)| \geq R \Rightarrow|F(z)| \geq C|f(z)|^{q} .
$$

The basic characterisation of separation at infinity is given in [20].

Proposition 7.1 ([20, Proposition 1.1]). Let $F: \mathbb{C}^{n} \rightarrow \mathbb{C}^{m}$ be a polynomial mapping and let $f: \mathbb{C}^{n} \rightarrow \mathbb{C}$ be a polynomial. Then the following conditions are equivalent:

(i) $F$ and $f$ are separated at infinity,

(ii) $\{0\} \times \mathbb{C} \not \subset \overline{(F, f)\left(\mathbb{C}^{n}\right)}$,

(iii) there is a polynomial $P: \mathbb{C}^{m} \times \mathbb{C} \rightarrow \mathbb{C}$ such that $P(F, f)=0$ and $\left.P\right|_{\{0\} \times \mathbb{C}} \neq 0$.

We shall say that $F$ and $f$ are separated in a neighbourhood of the fibre $f^{-1}(\lambda)$, where $\lambda \in \mathbb{C}$, if there exist $C, \varepsilon>0$ and $q \in \mathbb{R}$ such that

$$
|f(z)-\lambda| \leq \varepsilon \Rightarrow|F(z)| \geq C|f(z)|^{q} .
$$

From Proposition 7.1, it is easy to see that the above two definitions of separation are equivalent. Namely we have 
Proposition 7.2. Let $F: \mathbb{C}^{n} \rightarrow \mathbb{C}^{m}$ be a polynomial mapping and let $f: \mathbb{C}^{n} \rightarrow \mathbb{C}$ be a polynomial. Then the following conditions are equivalent:

(i) $F$ and $f$ are separated at infinity,

(ii) $F$ and $f$ are separated in a neighbourhood of the fibre $f^{-1}(0)$,

(iii) $F$ and $f$ are separated in a neighbourhood of any fibre $f^{-1}(\lambda), \lambda \in \mathbb{C}$.

According to Proposition 7.2, we shall call $F$ and $f$ separated if $F$ and $f$ are separated at infinity or in a neighbourhood of the fibre of $f$.

Let us pass to a separation condition for the gradient.

Proposition 7.3. Let $f: \mathbb{C}^{n} \rightarrow \mathbb{C}$ be a polynomial and let $d=\operatorname{deg} f$ $>0$. Then the following conditions are equivalent:

(i) $\operatorname{grad} f$ and $f$ are separated,

(ii) there exist $R, C>0$ such that

$$
|f(z)| \geq R \Rightarrow|\operatorname{grad} f(z)| \geq C|f(z)|^{-(d-1)^{n}},
$$

(iii) for any $\lambda \in \mathbb{C}$, there exist $C, \varepsilon>0$ such that

$$
|f(z)-\lambda| \leq \varepsilon \Rightarrow|\operatorname{grad} f(z)| \geq C|f(z)-\lambda|^{(d-1)^{n}},
$$

The above proposition is a generalisation of Theorem 2 in [25]. The proof will be preceded by a lemma.

LEMmA 7.4. Let $f: \mathbb{C}^{n} \rightarrow \mathbb{C}$ be a polynomial with $d=\operatorname{deg} f>1$. If grad $f$ and $f$ are separated, then there exists a polynomial $P \in \mathbb{C}\left[y_{1}, \ldots, y_{n}, t\right]$ such that $P(\operatorname{grad} f, f)=0,\left.P\right|_{\{0\} \times \mathbb{C}} \neq 0$ and $\operatorname{deg}_{t} P \leq(d-1)^{n}$.

Proof. We shall use the method developed in [18] (see proof of the main result). Let $V=\overline{(\operatorname{grad} f, f)\left(\mathbb{C}^{n}\right)} \subset \mathbb{C}^{n} \times \mathbb{C}$ and $k=\operatorname{dim} V$. Obviously $k \leq n$. Then, by Proposition 6.1, \{0\} $\times \mathbb{C} \not \subset V$. Without loss of generality, we may assume that $(0,0) \notin V$. Then there exists a linear mapping $L: \mathbb{C}^{n} \times \mathbb{C} \rightarrow$ $\mathbb{C}^{k+1}$ such that $\left.L\right|_{V}$ is a proper mapping and $L(0,0)=0 \notin L(V)$. After composition of $L$ with some linear automorphism $\mathbb{C}^{k+1} \rightarrow \mathbb{C}^{k+1}$ we may assume that for $G=L \circ(\operatorname{grad} f, f)$ we have $\operatorname{deg} g_{i} \leq d-1, i=1, \ldots, k$, and $\operatorname{deg} g_{k+1} \leq d$, where $G=\left(g_{1}, \ldots, g_{k+1}\right)$. Thus there exists a polynomial $\widetilde{P}: \mathbb{C}^{k+1} \rightarrow \mathbb{C}$ such that $L(V)=\left\{y \in \mathbb{C}^{k+1}: \widetilde{P}(y)=0\right\}$ and $\widetilde{P}(0) \neq 0$.

It is easy to see that there exists an affine subspace $M \subset \mathbb{C}^{n}$ with $\operatorname{dim} M=k$ such that $V=\overline{(\operatorname{grad} f, f)(M)}$, so $L(V)=\overline{G(M)}$. In consequence, by the Perron Theorem ([16, Satz 57]) there exists a nonzero polynomial $Q \in \mathbb{C}\left[y_{1}, \ldots, y_{k+1}\right]$ of the form

$$
Q=\sum_{\left(\nu_{1}+\cdots+\nu_{k}\right)(d-1)+\nu_{k+1} d \leq(d-1)^{k} d} c_{\nu_{1}, \ldots, \nu_{k+1}} y_{1}^{\nu_{1}} \cdots y_{k}^{\nu_{k}} y_{k+1}^{\nu_{k+1}}
$$

such that $Q\left(g_{1}, \ldots, g_{k+1}\right)=0$. Since $\operatorname{dim} L(V)=k$ and $\left.Q\right|_{L(V)}=\left.\widetilde{P}\right|_{L(V)}=0$, the polynomials $Q$ and $\widetilde{P}$ have a common divisor $R$ such that $R(G)=0$. 
Moreover, by the definition of $Q, \operatorname{deg}_{y_{k+1}} R \leq \operatorname{deg}_{y_{k+1}} Q \leq(d-1)^{n}$. Putting $P=R \circ L: \mathbb{C}^{n} \times \mathbb{C} \rightarrow \mathbb{C}$ we easily get the assertion.

Proof of Proposition 7.3. If $d=1$, then the assertion is trivial. Assume that $d>1$.

Assume that (i) holds. By Lemma 7.4 there exists a polynomial $P \in$ $\mathbb{C}\left[y_{1}, \ldots, y_{n}, t\right]$ such that $P(\operatorname{grad} f, f)=0, P_{\{0\} \times \mathbb{C}} \neq 0$ and $\operatorname{deg}_{t} P \leq(d-1)^{n}$. Hence, by Lemma 3.1 in [19], we get (ii). By using an analogous method, we shall prove (iii). Take any $\lambda \in \mathbb{C}$ and put $\widetilde{P}\left(y_{1}, \ldots, y_{n}, t\right)=P\left(y_{1}, \ldots, y_{n}, t+\lambda\right)$. Then $\widetilde{P}(\operatorname{grad} f, f-\lambda)=0$ and $\left.\widetilde{P}\right|_{\{0\} \times \mathbb{C}} \neq 0$. If $\widetilde{P}(0) \neq 0$, then (iii) is obvious. Assume that $\widetilde{P}(0)=0$. Then $P$ is $t$-regular. Thus, by the Weierstrass Preparation Theorem, there exist neighbourhoods $\Omega=\left\{y \in \mathbb{C}^{m}:|y| \leq \eta\right\}, \Delta=$ $\{t \in \mathbb{C}:|t| \leq \varepsilon\}, \eta, \varepsilon>0$, of the origins and a distinguished pseudopolynomial $g$ in $t, 0<N=\operatorname{deg}_{t} g \leq(d-1)^{n}$ of the form $g=t^{N}+g_{1} t^{N-1}+\cdots+g_{N}$, where $g_{i}: \Omega \rightarrow \mathbb{C}$ are holomorphic, $i=1, \ldots, N$, such that

$$
\{(y, t) \in \Omega \times \Delta: P(y, t)=0\}=\{(y, t) \in \Omega \times \Delta: g(y, t)=0\} .
$$

Diminishing $\eta$ and $\varepsilon$ if necessary, we may assume that $\varepsilon^{(d-1)^{n}} \leq \eta<1$. Then for any $|f(z)| \leq \varepsilon$, we have

$$
|f(z)| \leq 2 \max _{i=1, \ldots, N}\left|g_{i}(\operatorname{grad} f(z))\right|^{1 / i} \leq C_{1}|\operatorname{grad} f(z)|^{1 /(d-1)^{n}}
$$

for some $C_{1}>0$. This gives (iii). The implications (iii) $\Rightarrow($ i), (ii) $\Rightarrow$ (i) are obvious.

Let us give the main result of this section.

Theorem 7.5. Let $f: \mathbb{C}^{2} \rightarrow \mathbb{C}$ be a polynomial with $d=\operatorname{deg} f>0$. Then

(i) there exist $C, R>0$ such that

$$
|f(z)| \geq R \Rightarrow|\operatorname{grad} f(z)| \geq C|f(z)|^{-(d-1)^{2}},
$$

(ii) for any $\lambda \in \mathbb{C}$ there exist $C, \varepsilon>0$ such that

$$
|f(z)-\lambda| \leq \varepsilon \Rightarrow|\operatorname{grad} f(z)| \geq C|f(z)-\lambda|^{(d-1)^{2}} .
$$

Proof. By [25, Theorem 2] and Proposition 7.2 we know that the set $K_{\infty}(f)$ is finite if and only if $\operatorname{grad} f$ and $f$ are separated. Then by Corollary 5.2 and Proposition 7.3 we obtain the assertion.

Remark 7.6. As in Proposition 5.1 we may define the Fedoryuk condition $(\mathrm{F})$ for a polynomial $f$ in several variables. In this case the set of values for which (F) fails is also denoted by $K_{\infty}(f)$. By [25, Theorem 2] and Proposition 7.2 we conclude that $\operatorname{grad} f$ and $f$ are separated if and only if the set $K_{\infty}(f)$ is finite. 
REMARK 7.7. From Theorem 7.5 we deduce that for any real polynomial $f: \mathbb{R}^{2} \rightarrow \mathbb{R}$, grad $f$ and $f$ are separated.

Acknowledgements. We are deeply grateful to Jacek Chądzyński, Arkadiusz Płoski and Tadeusz Krasiński for their valuable comments and advice.

\section{References}

[1] J. Bochnak and S. Łojasiewicz, A converse of the Kuiper-Kuo theorem, in: Proc. Liverpool Singularities Symposium I (1969/70), Lecture Notes in Math. 192, Springer, Berlin, 1971, 254-261.

[2] Z. Charzyński and A. Kozłowski, Geometry of polynomials (II), Bull. Soc. Sci. Lett. Łódź 28 (1978), 1-10.

[3] J. Chądzyński and T. Krasiński, Exponent of growth of polynomial mappings of $\mathbb{C}^{2}$ into $\mathbb{C}^{2}$, in: Singularities, S. Eojasiewicz (ed.), Banach Center Publ. 20, PWN, Warszawa, 1988, 147-160.

[4] -, - A set on which the Eojasiewicz exponent at infinity is attained, Ann. Polon. Math. 67 (1997), 191-197.

[5] -, -, The gradient of a polynomial at infinity, Kodai Math. J. 26 (2003), 317-339.

[6] M. V. Fedoryuk, Asymptotic behaviour of the Fourier transform of the exponential of a polynomial, Dokl. Akad. Nauk SSSR 227 (1976), 580-583 (Russian); English transl.: Soviet Math. Dokl. (2) 17 (1976), 486-490.

[7] J. Gwoździewicz and A. Płoski, Formulae for the singularities at infinity of plane algebraic curves, Univ. Iagel. Acta Math. 39 (2001), 109-133.

[8] H. V. Ha, Nombres de Eojasiewicz et singularités à l'infini des polynômes de deux variables complexes, C. R. Acad. Sci. Paris 311 (1990), 429-432.

[9] Z. Jelonek and K. Kurdyka, On asymptotic critical values of a complex polynomial, J. Reine Angew. Math. 565 (2003), 1-11.

[10] P. Koebe, Über die Uniformisierung beliebiger analytischer Kurven, Göttinger Nachr. 1907, 198-207; 1909, 68-76.

[11] T. C. Kuo and Y. C. Lu, On analytic function germs of two complex variables, Topology 16 (1977), 299-310.

[12] T. C. Kuo and A. Parusiński, Newton polygon relative to an arc, in: J. W. Bruce and F. Tari (eds.), Real and Complex Singularities (São Carlos, 1998), 76-93.

[13] J. Milnor, Singular Points of Complex Hypersurfaces, Ann. of Math. Stud. 61, Princeton Univ. Press, Princeton, NJ, and Univ. of Tokyo Press, Tokyo, 1968.

[14] D. Mumford, Algebraic Geometry I. Complex Projective Varieties, Springer, Berlin, 1976.

[15] A. Parusiński, On the bifurcation set of complex polynomial with isolated singularities at infinity, Compos. Math. 97 (1995), 369-384.

[16] O. Perron, Algebra I. Die Grundlagen, de Gruyter, Berlin, 1932.

[17] F. Pham, La descente des cols par les onglets de Lefschetz, avec vues sur GaussManin, in: Systèmes différentiels et singularitiés, Juin-Juillet 1983, Astérisque 130 (1985), 11-47.

[18] A. Płoski, On the growth of proper polynomial mappings, Ann. Polon. Math. 45 (1985), 297-309.

[19] -, An inequality for polynomial mappings, Bull. Polish Acad. Sci. Math. 40 (1992), $265-269$. 
[20] A. Płoski and P. Tworzewski, A separation condition for polynomial mappings, ibid. 44 (1996), 327-331.

[21] P. J. Rabier, Ehresmann's fibrations and Palais-Smale conditions for morphisms of Finsler manifolds, Ann. of Math. 146 (1997), 647-691.

[22] T. Rodak, The Eojasiewicz exponent of the gradient in the ring of formal power series, preprint, 2002; Faculty of Mathematics Univ. of Łódź, http://www.math.uni. lodz.pl/preprints.

[23] S. Saks and A. Zygmund, Analytic Functions, PWN, Warszawa 1965.

[24] S. Smale, The fundamental theorem of algebra and complexity theory, Bull. Amer. Math. Soc. 4 (1981), 1-36.

[25] S. Spodzieja, Eojasiewicz inequality at infinity for the gradient of a polynomial, Bull. Polish Acad. Sci. Math. 50 (2002), 273-281.

[26] - Koebe covering theorem and singularities of holomorphic functions, in: Proc. XXIV Instructional Conf. in Complex Analytic and Algebraic Geometry, Łódź Univ. Press, 2003, 45-56 (in Polish).

Department of Mathematics

Technical University

Al. Tysiąclecia Państwa Polskiego 7

25-314 Kielce, Poland

E-mail: matjg@tu.kielce.pl
Faculty of Mathematics

University of Łódź

Banacha 22

90-238 Łódź, Poland

E-mail: spodziej@imul.uni.lodz.pl

Reçu par la Rédaction le 24.5.2004

Révisé le 5.10.2005 